\title{
The $k d r$-bearing haplotype and susceptibility to Plasmodium falciparum in Anopheles gambiae: genetic correlation and functional testing
}

Christian Mitri ${ }^{1 \dagger}$, Kyriacos Markianos ${ }^{2 \dagger}$, Wamdaogo M. Guelbeogo ${ }^{3}$, Emmanuel Bischoff $^{1}$, Awa Gneme ${ }^{3}$, Karin Eiglmeier ${ }^{1}$, Inge Holm ${ }^{1}$, N'Fale Sagnon ${ }^{3}$, Kenneth D. Vernick ${ }^{1,4^{*}}$ and Michelle M. Riehle ${ }^{4}$

\begin{abstract}
Background: Members of the Anopheles gambiae species complex are primary vectors of human malaria in Africa. It is known that a large haplotype shared between An. gambiae and Anopheles coluzzii by introgression carries point mutations of the voltage-gated sodium channel gene para, including the L1014F kdr mutation associated with insensitivity to pyrethroid insecticides. Carriage of L1014F $k d r$ is also correlated with higher susceptibility to infection with Plasmodium falciparum. However, the genetic mechanism and causative gene(s) underlying the parasite susceptibility phenotype are not known.

Methods: Mosquitoes from the wild Burkina Faso population were challenged by feeding on natural P. falciparum gametocytes. Oocyst infection phenotypes were determined and were tested for association with SNP genotypes. Candidate genes in the detected locus were prioritized and RNAi-mediated gene silencing was used to functionally test for gene effects on P. falciparum susceptibility.

Results: A genetic locus, Pfin6, was identified that influences infection levels of $P$. falciparum in mosquitoes. The locus segregates as a $\sim 3 \mathrm{Mb}$ haplotype carrying 65 predicted genes including the para gene. The haplotype carrying the $k d r$ allele of para is linked to increased parasite infection prevalence, but many single nucleotide polymorphisms on the haplotype are also equally linked to the infection phenotype. Candidate genes in the haplotype were prioritized and functionally tested. Silencing of para did not influence $P$. falciparum infection, while silencing of a predicted immune gene, serine protease ClipC9, allowed development of significantly increased parasite numbers.

Conclusions: Genetic variation influencing Plasmodium infection in wild Anopheles is linked to a natural $\sim 3$ megabase haplotype on chromosome $2 \mathrm{~L}$ that carries the $k d r$ allele of the para gene. Evidence suggests that para gene function does not directly influence parasite susceptibility, and the association of $k d r$ with infection may be due to tight linkage of $k d r$ with other gene(s) on the haplotype. Further work will be required to determine if ClipC9 influences the outcome of P. falciparum infection in nature, as well as to confirm the absence of a direct influence by para.
\end{abstract}

Keywords: Mosquito, Malaria, Host-pathogen interaction, Haplotype

\footnotetext{
*Correspondence: kvernick@pasteur.fr

${ }^{\dagger}$ Christian Mitri and Kyriacos Markianos contributed equally to this work

${ }^{4}$ Department of Microbiology, University of Minnesota, Saint Paul, MN

55108, USA

Full list of author information is available at the end of the article
}

\section{Ciomed Central}

(C) 2015 Mitri et al. This article is distributed under the terms of the Creative Commons Attribution 4.0 International License (http://creativecommons.org/licenses/by/4.0/), which permits unrestricted use, distribution, and reproduction in any medium, provided you give appropriate credit to the original author(s) and the source, provide a link to the Creative Commons license, and indicate if changes were made. The Creative Commons Public Domain Dedication waiver (http://creativecommons.org/ publicdomain/zero/1.0/) applies to the data made available in this article, unless otherwise stated. 


\section{Background}

Throughout sub-Saharan Africa, members of the Anopheles gambiae species complex are primary vectors of the human malaria parasite Plasmodium falciparum, which is responsible for extensive morbidity and mortality. Phenotypic differences for traits that influence malaria transmission, such as behaviour, Plasmodium susceptibility and insecticide resistance can be influenced by genetic variation [1-4].

Mosquito susceptibility to $P$. falciparum is variable and has a strong genetic component, as shown by populationbased mapping of quantitative trait loci $[3,5,6]$, laboratory-based phenotypic selection of resistant lines [7], and genetic association studies [8,9]. However, despite a genetic basis for variation in susceptibility to infection with $P$. falciparum, underlying causative single nucleotide polymorphisms (SNP) have neither been identified nor replicated in natural populations.

Similarly, a number of genetic mechanisms influence susceptibility to insecticides [10]. However, unlike the case of parasite susceptibility, individual SNPs have been robustly implicated. Increased resistance to pyrethroid insecticides is influenced in part by a SNP of the voltagegated sodium channel gene para (also called VSG). The mutation of amino acid residue 1014 of the conserved S6 transmembrane segment of para domain II from leucine to phenylalanine (L1014F) or serine (L1014S) is correlated with decreased excitability of the insect nervous system, which is thought to be related to phenotypic insensitivity to insecticide. The allele is named knockdown resistance $(k d r)$, and has arisen independently at the homologous position in An. gambiae [2] and other insects $[11,12]$. Other coding variants in and near para can also contribute to pyrethroid-insensitivity $[10,13]$. In An. gambiae, $\mathrm{L} 1014 \mathrm{~F}$ is referred to as $k d r$-W because it was originally described in West Africa, whilst L1014S, first described in East Africa, is called $k d r$-E $[14,15]$. The geographic labels however, are now misnomers as these resistance loci are not geographically restricted, because L1014F co-occurs with L1014S in East Africa [16] and rates of L1014S have been increasing in West Africa [17, $18]$.

Mosquito sister taxa Anopheles coluzzii and An. gambiae, the former $\mathrm{M}$ and $\mathrm{S}$ molecular forms, respectively [19], display different frequencies of the L1014F mutation. The L1014F allele is nearly fixed in An. gambiae in West Africa but segregates in many populations of $A n$. coluzzii as a consequence of introgression from An. gambiae to An. coluzzii occurring within the past $10-15$ years [18, 20-23]. Genotype analysis indicates that the introgression of kdr L1014F from An. gambiae to An. coluzzii occurred upon an extended chromosome haplotype [24, 25].
Recent studies have reported a correlation between $P$. falciparum susceptibility and the $k d r$ L1014F allele [26, 27]. However, para is just one among many genes carried on an extended haplotype, and consequently the $k d r$ L1014F SNP variant is a marker not only for propensity to pyrethroid resistance, but equally tags a block of up to 65 linked genes. There are no reports yet on which genes within this haplotype influence parasite susceptibility, or whether particular genes, for example para, might display both phenotypes both for parasite and insecticide resistance. The power to genetically dissect the phenotypes by fine-mapping will likely be diminished by the expected low frequency of recombination due to the haplotype structure, which will be further decreased by the centromeric location.

Here, significant genetic association of the para-bearing haplotype with susceptibility to wild $P$. falciparum was detected. Because of the limited power of recombinational fine-mapping to distinguish gene phenotypes within the locus, a candidate gene approach was used. Genes in the locus were prioritized by bio-informatic prediction of function. Two chosen candidates were silenced by RNAi and challenged with P. falciparum. A candidate gene with strong immune functional prediction, ClipC9, influenced parasite infection, while silencing of para did not change parasite infection levels. As with any functional testing of candidate genes, both of these observations require further confirmation.

\section{Methods \\ Mosquito sampling and wild Plasmodium falciparum infection}

Mosquitoes were sampled as larvae using the standard dipping method as previously described [28]. In order to collect a random sample of essentially unrelated individuals, fewer than ten larvae were collected from any one larval site and a single collection comprised larvae from $>50$ larval sites. Collections were done in the Sudan Savanna region of Burkina Faso in the village of Goundry $\left(12^{\circ} 30^{\prime} \mathrm{N}, 1^{\circ} 20^{\prime} \mathrm{W}\right), 30 \mathrm{~km} \mathrm{~N}$ of the capital city, Ouagadougou, across months of the malaria transmission season of 2007 and 2008 [29]. Larvae were raised in Centre National de Recherche et de Formation sur le Paludisme (CNRFP) insectaries in Ouagadougou to adulthood under standard insectary conditions [29]. Following emergence, 3-day old adults were challenged with wild P. falciparum by experimental infection. Feeding was done on an artificial membrane in a water-jacketed feeding device as described previously using gametocytaemic blood obtained from study participants [29]. Unfed mosquitoes were excluded from analysis and infection levels for fed mosquitoes were determined by counting midgut oocysts 7-8 days post infection. Genomic 
DNA was extracted from carcasses for SNP genotyping. Population sub-group composition was determined by published molecular diagnostic assays [28, 30]. Mosquitoes used for genetic association analysis came from five successful experimental infections on wild gametocytes using criteria defined previously [6, 28, 29, 31]: oocyst infection prevalence $\geq 30 \%$ and oocyst intensity in at least one mosquito in the group of $\geq 10$ oocysts. These criteria were applied to the mosquitoes raised together as a cohort and fed on the same blood, and identified the experiments with broad phenotype distributions. This infection-quality filter used for infection with wild gametocytes, which are inherently variable and noisy, assures that all analysed individuals came from an infection session with the power to distinguish between levels of susceptibility, free from confounding noise due to uninfective blood, technical failure or related factors. Low prevalence infections in the mosquitoes fed on gametocytaemic blood can potentially result from multiple causes, but regardless the cause, such groups are not informative for genetic analysis because of poor phenotype distribution. Mosquito genetics can be excluded as a cause, because the fed cohort is comprised of random unrelated individuals.

\section{SNP genotyping and genetic association}

DNA samples from dissected mosquito carcasses were subjected to whole genome amplification (WGA) (Genomiphi, GE Health Sciences) using supplied protocols. Following WGA, DNA was ethanol precipitated, concentrations determined by the picogreen method [32], and 500 ng was used for SNP genotyping by the Illumina GoldenGate method [33] by the Boston Children's Hospital Molecular Genetics Core Facility (IDDRC). The SNPs and their primer sequences are available in Additional file 1: Table S1. Data were analysed using BeadStudio software [33]. Genotypes of mosquitoes for the Pfin6/para haplotype were independently verified using the Sequenom MassArray method, with a set of seven diagnostic SNPs at the following genomic positions in the PEST reference genome: 2L.1272741 [T/C], 2L.1834476 [A/G], 2L.1970368 [C/T], 2L.2081228 [T/C], 2L.2430786 [C/T], 2L.2489023 [G/A], 2L.2489212 [T/C]. As these seven SNPs are in perfect linkage disequilibrium, they are all equally informative and thus comprise a sevenfold redundant assay for a single genotype feature, that is, the haplotype they are carried upon. Because the seven SNPs are all completely cross-informative for the haplotype, additional markers in this region would not increase resolution of the feature. For this, aliquots of the same DNA samples used for Illumina genotyping were genotyped using Sequenom at the University of Minnesota Genomic Center (UMGC). Sequenom genotypes were analysed for genetic association with malaria infection phenotype using the Haploview program [34].

\section{Gene silencing and functional assays}

A colony of An. coluzzii mosquitoes (Fd03) was initiated with the eggs of ten mated, gravid females captured in village houses in Goundry, Burkina Faso. The colony is wild-type for para and is maintained using standard laboratory conditions at the Institut Pasteur. Doublestranded RNAs (dsRNA) were synthesized from PCR amplicons using the T7 Megascript Kit (Ambion) as described previously [35]. The sequences of the primers used for amplification of ClipC9 dsRNA were:

T7_CLIPC9F:TAATACGACTCACTATAGGACCG TGCTGCAGAATGACTGC T7_CLIPC9R: TAATACG ACTCACTATAGGTATCCCTAGTAGCACTAACCG.

The primers used for amplification of the para/VSC dsRNA were:

T7-4707F:TAATACGACTCACTATAGGAGGGCTAT CCGGGAAATTGTGG.

T7-4707 R:TAATACGACTCACTATAGGTGAAGCG TCTGTTCCGCCTCC.

The underlined portion of the primer sequence is the T7 tag. For each targeted gene, $200 \mathrm{ng}$ of dsRNA (but not more than $200 \mathrm{nl}$, depending on the concentration) were injected into the thorax of cold-anesthetized 1-day old An. coluzzii females using a microinjector (Nanoject II, Drummond). Validation of gene silencing efficiency was done by RT-PCR. In all cases, treatment with dsRNA led to decreases in target transcript abundance compared to dsGFP-injected controls (Additional file 2: Figure S1). The same numbers of mosquitoes were treated with control and experimental dsRNA in each replicate.

For gene silencing assays, mosquitoes were challenged with $P$. falciparum strain NF54 gametocytes, cultured using an automated tipper-table system implemented in the CEPIA mosquito infection facility of the Institut Pasteur, as previously described [35]. For experimental infection, $10 \mathrm{ml}$ of medium containing mature gametocytes was centrifuged at $2000 \mathrm{rpm}$, and the cell pellet was resuspended in an equal volume of normal type $A B$ human serum. The infected erythrocytes were added to fresh erythrocytes in $A B$ human serum and transferred to a membrane feeder warmed to $37{ }^{\circ} \mathrm{C}$. Mosquitoes were allowed to feed for $15 \mathrm{~min}$. Unfed mosquitoes were removed, so that only fully engorged females were used for further analysis. Mosquitoes were maintained on $10 \%$ sucrose solution supplemented with $0.05 \%$ para-amino benzoic acid. At 7-8 days after the malaria infection challenge, all surviving mosquitoes were dissected. Thus, the dissected number, given as sample size (n) values below infection histograms, indicates survivorship, and control versus treatment values are compared as an estimate 
of gene-specific or overall mortality effects. At least two independent replicate infections were performed per condition. Replicates were analysed independently using the tests described below. If at least one replicate met the significance criterion of $\mathrm{p} \leq 0.05$, a third replicate was done.

\section{Analysis of infection phenotypes}

For infections on either wild or cultured gametocytes, infection phenotype was determined at 7-8 days post blood meal. Mosquito midguts were dissected, stained with dibromofluorescein (Sigma Chemical) and the number of midgut oocysts determined by light microscopy. For mosquitoes fed on wild gametocytes, infection prevalence was measured (the fraction of mosquitoes with at least one oocyst), while for functional genomic tests using mosquitoes fed on cultured gametocytes, two infection phenotypes were measured, infection prevalence, and oocyst intensity, counts of oocysts in only those individuals with $\geq 1$ oocyst. Oocyst intensity comparisons were not used for wild gametocyte infections because the sample size of mosquitoes with $\geq 1$ oocyst did not provide sufficient statistical power for intensity.

For statistical analysis, comparisons of infection prevalence were made using the Chi Square test, and comparisons of oocyst intensity using the non-parametric Wilcoxon Mann-Whitney test. Replicates were analysed independently using the tests described above and $\mathrm{p}$ values from independent tests of significance were combined using the meta-analytical approach of Fisher [36], and this combined $\mathrm{p}$ value is reported here. The threshold for significance was defined as $\mathrm{p}=0.01$.

\section{Ethical considerations}

For collection of blood from $P$. falciparum gametocyte carriers for experimental membrane feeder infection of mosquitoes, the study protocol was reviewed and approved by the institutional and national health ethical review board (Commission Nationale d'Ethique en Santé) of Burkina Faso (code No 2006-032). The study procedures, benefits and risks were explained to parents or legal guardians of children and their informed consent was obtained. Children of parents or guardians who had given consent were brought to CNRFP the day of the experiment for gametocyte carrier screening. All children were followed and symptomatic subjects were treated with the combination of artemether-lumefantrine $\left(\right.$ Coartem $\left.^{\circledR}\right)$ according to relevant regulations of the Burkina Faso Ministry of Health.

\section{Results}

\section{Structure of the para gene locus}

Wild mosquitoes were collected as larvae in Burkina Faso, were reared to adults in the insectary, and were challenged with wild $P$. falciparum parasites by membrane feeding on blood from gametocyte carriers, yielding 363 mosquitoes belonging to the An. gambiae species complex that were used in the study.

The mosquito DNAs were genotyped with 105 SNPs spanning a broad $\sim 15 \mathrm{Mb}$ region containing the para gene across the centromere of chromosome 2, between coordinates chromosome $2 \mathrm{R}: 55 \mathrm{Mb}$ to chromosome 2L:10 Mb (Additional file 1: Table S1; Fig. 1, bottom track of 105 SNPs shown as black vertical lines). Analysis of the individual mosquito genotypes reveals a region of elevated local homozygosity that defines the boundaries of two allelic haplotypes, termed HapA and HapB (Fig. 1). When mosquitoes were categorized by their HapA/HapB genotype, homozygotes are evident as regions of high linkage, with additional unlinked variation seen as dips in homozygosity (Fig. 1, homozygosity plot for each genotype). The core haplotype across both alleles is $\sim 3 \mathrm{Mb}$ in size (Fig. 1, blue horizontal lines superimposed on red homozygosity plot). This corresponds to the location and estimated $3.3 \mathrm{Mb}$ size of the haplotype bearing the $k d r$ allele of the para gene that was reported as an introgression from An. gambiae to An. coluzzii [24, 25].

The current sample set was comprised of three population groups of the An. gambiae species complex ( $A n$. gambiae, $\mathrm{n}=46$, An. coluzzii, $\mathrm{n}=53$, and Goundry form, $\mathrm{n}=264$ ). The Goundry form is a population sub-group within An. gambiae sensu lato with unknown taxonomic status or epidemiology for malaria transmission [28, 37, 38]. The haplotype structure of the para gene region in Goundry was not previously known. Of the 105 genotyped markers in this region, a sub-set of 26 SNPs span the haplotype (Fig. 1, middle track of SNPs shown as purple vertical lines) and were used to examine segregation of haplotype alleles across the three taxa. Consistent with observations that the $k d r$ mutation introgressed from $A n$. gambiae to An. coluzzii upon a haplotype [20, 21, 24, 25], the 26 SNPs in this genomic region did not distinguish between An. gambiae, An. coluzzii and the Goundry form (Fig. 2a). This result indicates that the para-bearing haplotype is identical by descent (IBD) across the three taxa, with strong ancestral sharing as well as the acquisition of additional mutations over time.

\section{Genetic association analysis for Plasmodium falciparum susceptibility}

Tests for genetic association are done within panmictic population groups to avoid stratification artifacts [39]. Of the three population groups in the current study, $A n$. gambiae have no power to test for association because the $k d r$ allele is essentially fixed in An. gambiae, consistent with other reports [20,21], and despite segregation of the $k d r$ allele within An. coluzzii, the sample size was 


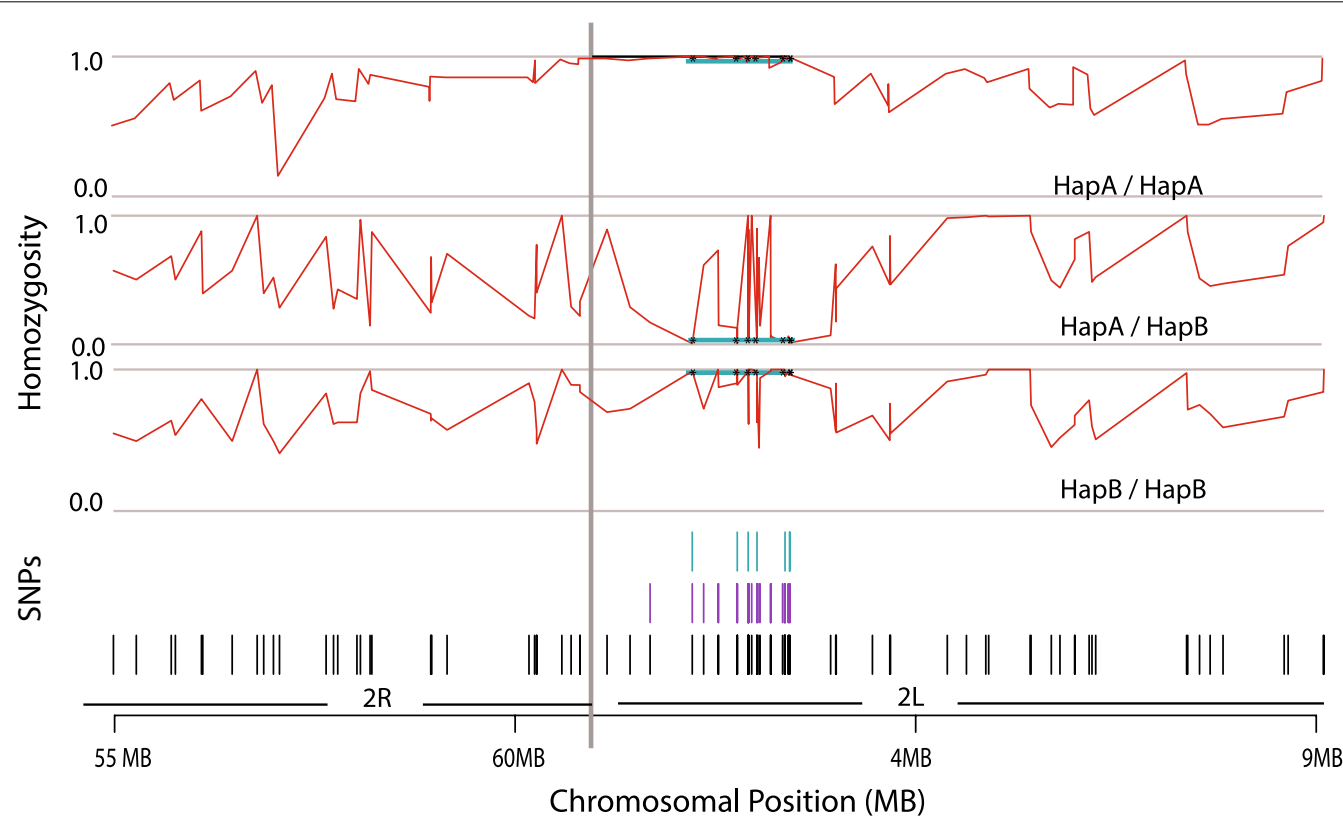

Fig. 1 Fine structure of haplotypes in the para gene region measured as level of homozygosity. Mosquitoes were genotyped across $\sim 15 \mathrm{Mb}$ spanning the chromosome 2 centromere (Iong vertical grey line), first using a set of 105 SNPs (black SNP track) to detect the extent and approximate limits of the haplotype containing the para gene. Individuals were then grouped based on their genotype as defined by seven highly informative haplotype-tagging markers (blue SNP track), yielding three groups with haplotype status, HapA/HapA, HapA/HapB, HapB/HapB. Homozygosity was plotted for all individuals by genotype, depicted on the $y$ axis as the fraction of homozygous individuals at each marker nucleotide position (for all individuals homozygous, $y=1$; for all individuals heterozygous, $y=0$ ). The region of homozygosity for the haplotype-tagging markers is indicated by a horizontal blue line, with the seven tag SNP positions marked by black stars within the horizontal blue line. The HapA allele carries the kdr mutation of the para gene

insufficient for an adequately powered statistical test. Thus, in the current study, only the Goundry group sample was appropriate for an association test of the parabearing haplotype. As shown (Fig. 2A), a haplotype with a common evolutionary origin segregates in all three population groups. Thus, although the genetic background may differ among taxa, the same para-bearing haplotype is present in all of these taxa, and tests for association of the haplotype with $P$. falciparum infection levels were performed within the Goundry group.

A sub-set of seven completely correlated SNPs were chosen as haplotype-tagging markers for genetic association (Fig. 1, top track of SNPs shown as blue vertical lines). The seven SNPs mark ancestral differentiation, are in perfect linkage disequilibrium (LD) (thus are redundant tests for the same feature), and efficiently tag HapA and HapB alleles. Association of the para-bearing haplotype with susceptibility to $P$. falciparum was tested in the Goundry sub-group mosquitoes using the haplotypetagging SNPs combined as a single redundant assay for the haplotype. Mosquitoes homozygous for haplotype allele HapA were more susceptible to infection with $P$. falciparum than heterozygotes or HapB homozygotes $(p=0.0293$ for oocyst infection prevalence; Fig. 2B). The locus was named Plasmodium falciparum infection locus 6 (Pfin6) following naming convention [31].

\section{Functional dissection of the Pfin6 locus}

The haplotype that carries the mapped Pfin6 locus as well as the para gene is $\sim 3.3 \mathrm{Mb}$ in length $[24,25]$ and contains 65 predicted genes (Fig. 2C; Additional file 3: Table S2). Because the haplotype contains long blocks of SNPs in perfect linkage, resolution by recombinational finemapping is not expected to be productive. Fine-mapping would be further complicated by the centromeric location of the mapped locus, as centromeres typically have lower than average recombination rates. Instead, in the present study a candidate gene approach is employed, dissecting the locus by functional testing of candidates ascertained by predicted gene function and other evidence. It is important to note that candidate gene assays can provide suggestive evidence, but do not constitute proof of genetic causation.

Of the genes in the locus (AGAP004677-AGAP004742), two are potentially involved in host-defense processes: AGAP004719 (2L:2.71 Mb), with strong prediction of immune function, identified as ClipC 9 , a clip-domain serine protease homologous with the Drosophila 

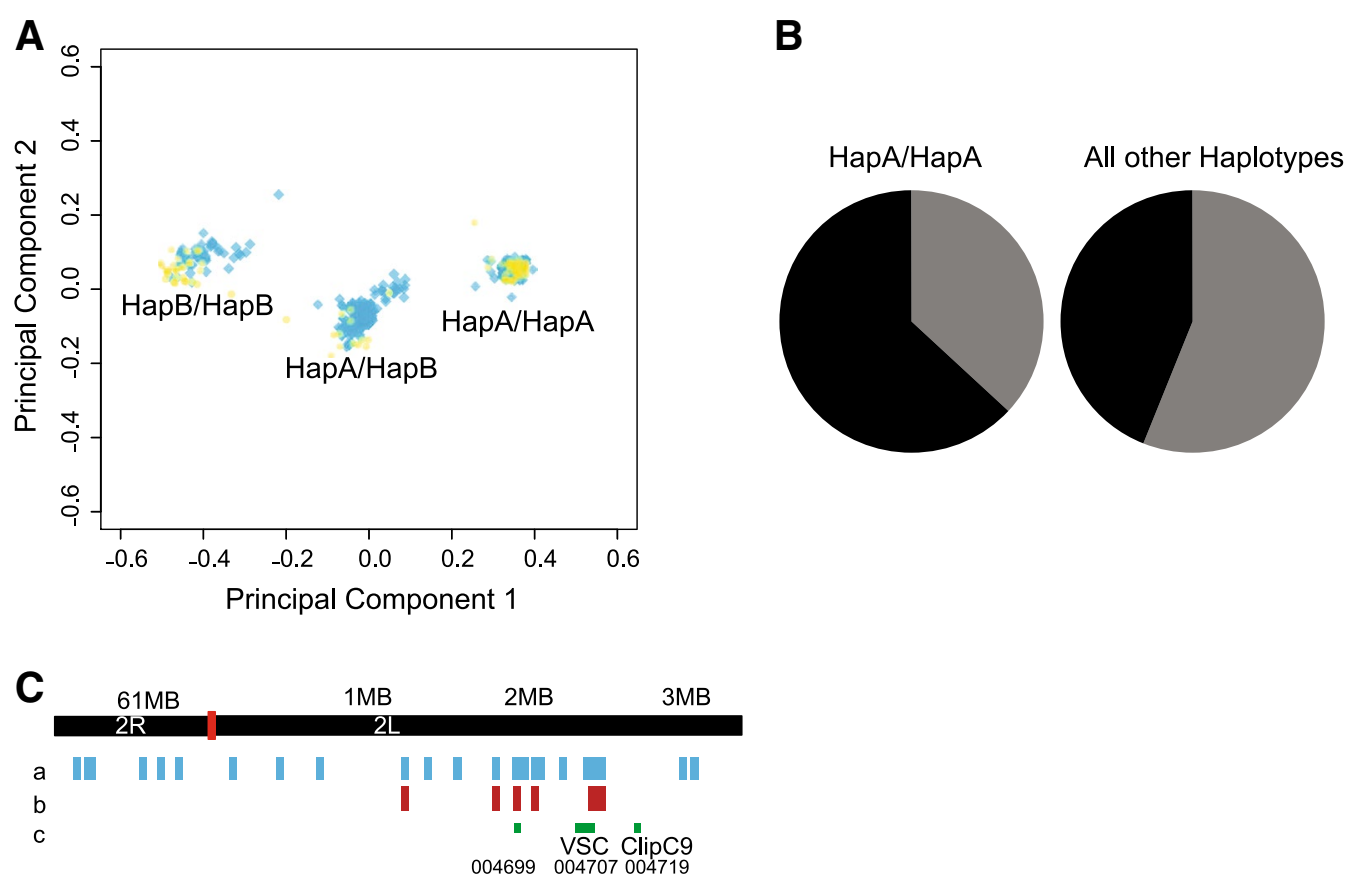

Fig. 2 Population genetic association and analysis of haplotype locus. A Genetic markers in the para gene region of chromosome $2 \mathrm{~L}$ clearly genotype haplotype alleles but do not distinguish between different population subgroups. Strong ancestral sharing of IBD genetic haplotypes across population subgroups indicates a shared common origin of the haplotype. An. coluzzii and An. gambiae (yellow circles), and Goundry form (blue squares), are shown on the PCA plot generated using the extended set of 26 haplotype-tagging SNPs (purple SNP track in Fig. 1). B Wild-collected individuals of the Goundry sub-group homozygous for HapA $(n=84)$ were significantly more susceptible to infection with natural P. falciparum than mosquitoes with any other haplotype $(n=180)$. Pie chart indicates mosquitoes infected with P. falciparum (black), and the uninfected mosquitoes (grey) after feeding together on blood from naturally infected donors. Unfed mosquitoes were removed from the experiment, thus all analysed mosquitoes ingested infectious parasites. C Pfin6 candidate genes indicated by Vectorbase AGAP gene ID, with positions of the functionally-tested genes ClipC9 and paraNSC shown

extracellular serine protease Persephone (CG6367), which mediates activation of the Toll signalling pathway in response to fungal infection [40]; and AGAP004699 (2L:1.97 Mb), weakly predicted as potentially immune, which codes for a serine/threonine-protein kinase with a Drosophila orthologue involved in haemocyte differentiation (Raf homologue serine/threonine-protein kinase phl, CG2845, [41]. The locus also contains AGAP004707 ( $2 \mathrm{~L}: 2.35 \mathrm{Mb})$, the para gene in which the $k d r$ mutant is associated with response to pyrethroid insecticides.

The following lines of evidence prioritized the functional testing of candidates ClipC9/AGAP004719 and para/AGAP004707 for their effect on the outcome of $P$. falciparum infection, using RNAi-mediated gene silencing assays. ClipC 9 is predicted to be catalytically active as a serine protease, bearing all cysteine residues involved in disulfide bonds, as well as the conserved residues of the catalytic triad. Expression of ClipC9 was upregulated following infection with Gram-negative bacteria and down-regulated following infection with Gram-positive bacteria [42, 43], but was unchanged following infection with Plasmodium [43]. The para gene would not be selected by the same criteria as a candidate for functional testing, because it does not display any known or predicted immune function, but it was chosen on an ad hoc basis because of reports of association of $k d r$ with infection $[26,27]$. The para gene influence upon any phenotype has not been functionally analysed by gene silencing, according to published reports.

Mosquitoes from an $A n$. coluzzii colony recently initiated from the Burkina Faso study site were injected with double-stranded RNA (dsRNA) targeting ClipC9, para, or control GFP. Four days after dsRNA treatment, decreased transcript levels were verified (Additional file 2: Figure S1), mosquitoes were fed on cultured $P$. falciparum gametocytes, and 7-8 days later midgut oocysts were counted. Infection intensity (numbers of oocysts in mosquitoes with $\geq 1$ oocyst) was consistently higher in the dsClipC9-treated individuals as compared to controls (Fig. 3; $\mathrm{p}=6.23 \times 10^{-4} ; \mathrm{p}$ values from three replicate experiments combined using the Fisher Method; individual $p$ values $0.005,0.089,0.017)$. There was no effect of dsClipC9 treatment upon infection prevalence (the 

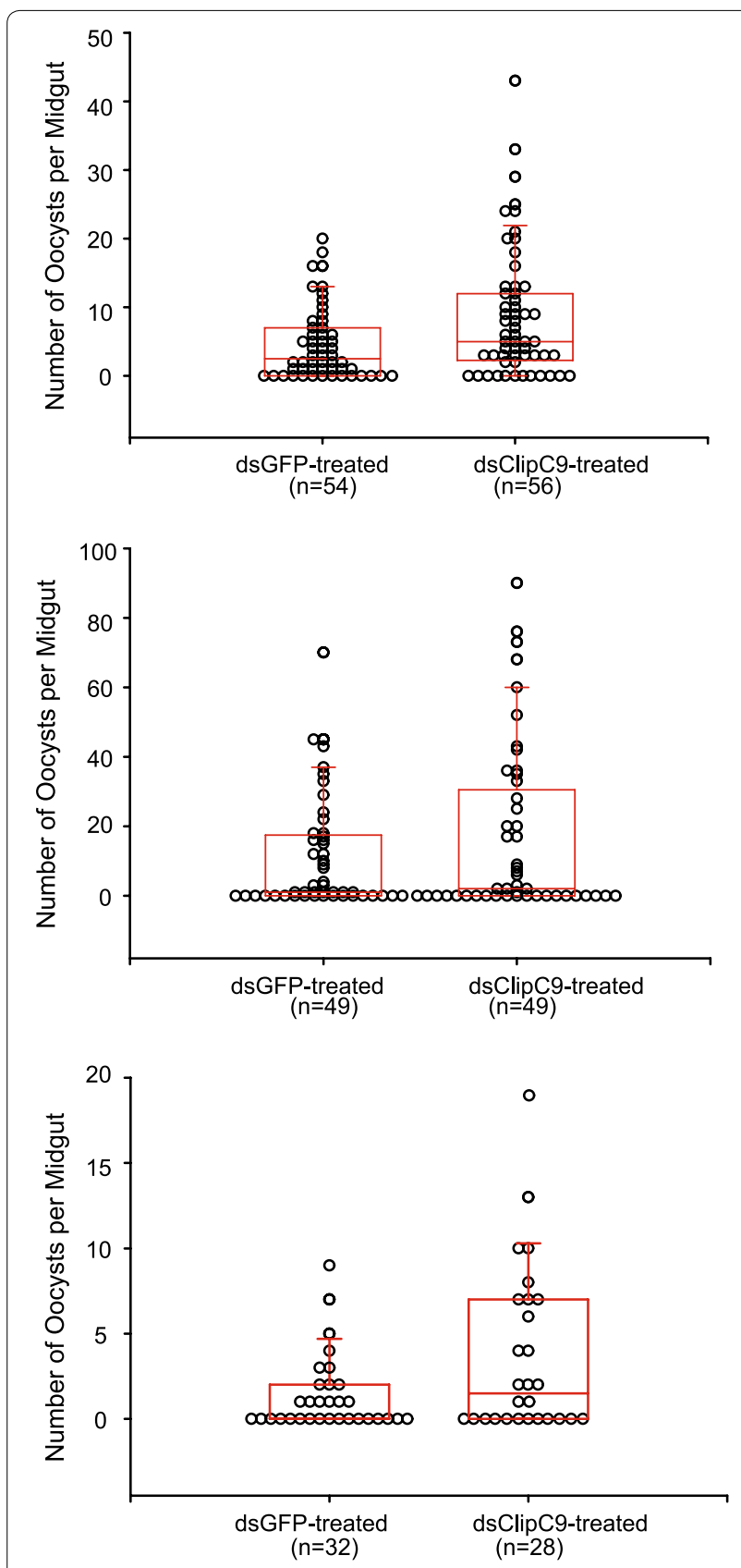

Fig. 3 Candidate gene ClipC9 within the Pfin6 locus protects mosquitoes against infection with Plasmodium falciparum. Expression of the ClipC9 (AGAP004719) gene was silenced by dsRNA injection, mosquitoes were challenged with cultured $P$. falciparum gametocytes, and infection outcomes were compared with control mosquitoes treated with dsGFP. In all three replicate experiments, the dsClipc9-treated mosquitoes permitted development of significantly higher numbers of midgut oocysts as compared to controls (combined $p$ value, $5.16 \times 10^{-4}$; three replicate experiments are shown with box plots, $p$ values from individual experiments were combined using the Fisher Method). Thus, the activity of ClipC9 influences $P$. falciparum oocyst infection intensity in A. gambiae. There was no difference in oocyst infection prevalence between ClipC9-silenced mosquitoes and GFP controls ( $p=0.78)$ proportion of mosquitoes with $\geq 1$ oocyst, $\mathrm{p}=0.78$; individual $\mathrm{p}$ values $0.658,0.678,0.450$ ).

For the para voltage-gated sodium channel (VSC) gene, there was no statistically significant difference for either infection prevalence or intensity between ds VSC-treated as compared to control mosquitoes (infection prevalence $\mathrm{p}=1.0$, infection intensity $\mathrm{p}=0.10$, Fig. 4). Thus, under these conditions, abolishing para gene transcriptional activity does not appear to influence parasite development, although the effect of transcript silencing on protein levels is not known.

Testing gene function by silencing, as shown here, is distinct from testing allele function by genetic association, as shown above. Gene silencing mediated by a $~ 500$ bp double-stranded RNA targeting the gene is immune to sequence differences greater than the $k d r$ SNP variant between para alleles [44]. The colony used for the functional test was fixed for wild-type para, and transcript silencing is efficient (Additional file 2: Figure S1). Thus, para gene transcripts are abolished by silencing, regardless of the genetic variants they carry.

The effect of gene silencing on mosquito mortality was monitored by comparison of control dsGFP and experimental dsRNA treatments. The same numbers of control and experimental mosquitoes were injected with dsRNA per replicate, and all surviving mosquitoes were dissected, thus the sample sizes (n values shown on Figs. 3, 4) indicate overall survival per treatment. There was no evidence of a consistent difference in survival between mosquitoes treated with dsClipC9 as compared to dsGFP controls (Fig. 3), or between dsVSC-treated as compared to dsGFP controls (Fig. 4). Thus, it appears that neither ClipC9 nor para gene activity is biologically essential for mosquito viability, although minor effects on survival cannot be ruled out.

\section{Discussion}

The Pfin6 malaria infection locus and $k d r$ are carried on the same haplotype

Results presented here show that a $\sim 3 \mathrm{Mb}$ haplotype is shared by introgression as an identical by descent (IBD) feature among three otherwise reproductively isolated groups of the An. gambiae species complex. Two of the three groups, An. gambiae and An. coluzzii, display overall equivalent susceptibility to wild $P$. falciparum [29, $45-48$ ], while the Goundry form is more susceptible to experimental infection with wild parasites [28]. However, because the haplotype is a shared feature with common evolutionary origin among the three population groups, it is unlikely to explain this phenotypic difference between the groups.

The unit of introgression of the $k d r$ allele was not simply the $k d r$ variant nucleotide, but rather an extended 


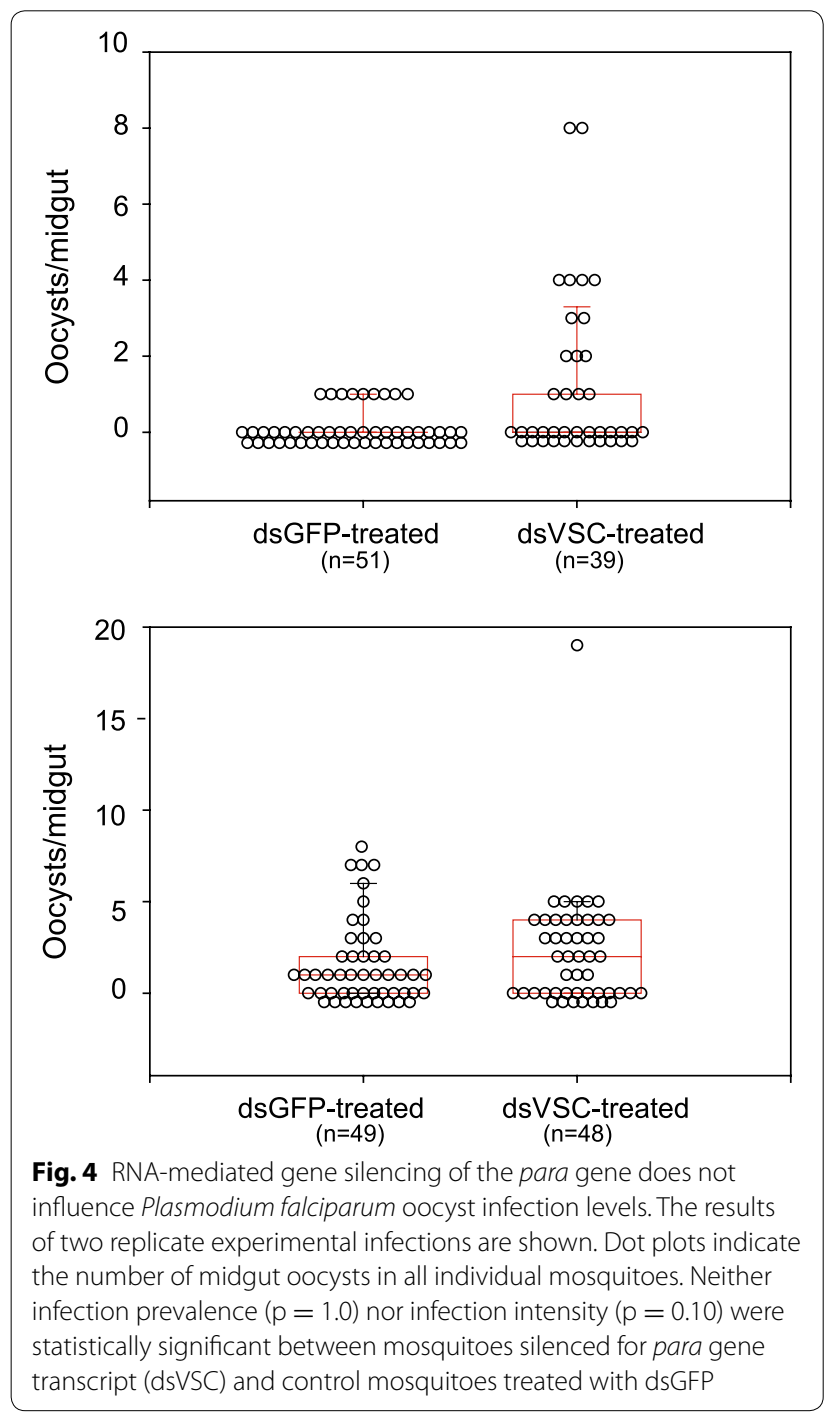

haplotype bearing para and $>60$ other neighbouring genes. The $\sim 3 \mathrm{Mb}$ size of the introgressed haplotype reported here is consistent with other reports [24, 25]. It has been suggested that exposure to insecticides used in agriculture or on bed nets could be driving the spread of $k d r$, based on the temporal correlation of the two events $[23,49,50]$. The finding that the pathogenresistance locus, Pfin6, maps to the same haplotype suggests a potential complementary explanation for the adaptive introgression of this haplotype. Components of the immune systems of An. coluzzii and An. gambiae mosquitoes are under distinct selective pressure that has strongly altered the alleles and diversity at particular immune loci unrelated to Pfin6 [37, 51, 52]. In those cases, it is hypothesized that exposure to distinct pathogen profiles in the different ecological niches of $A n$. coluzzii and An. gambiae mosquitoes could be the source of this strong recent selection. The presence of a functional immunity locus upon this haplotype might explain at least part of the introgression pressure based on differential immunity to environmental microbes.

\section{Functional genomic analysis of the haplotype carrying Pfin6 and para}

Based on prediction of potential immune function one high-priority candidate gene was identified within the Pfin6 locus, the clip domain serine protease, ClipC9. This does not rule out that a different or multiple genes could actually underlie the Plasmodium susceptibility phenotype of Pfin6 detected in nature. ClipC 9 was functionally confirmed, by gene silencing, as a gene that influences mosquito infection levels with P. falciparum. Functional assays of candidate genes can demonstrate that a gene influences a similar phenotype to a genetically mapped locus, and thus could be a plausible genetic candidate. However, functional assays are not proof of genetic causation. Subsequent work will be required to establish whether naturally-occurring genetic variation in ClipC 9 underlies the genetic effect of the Pfin6 locus in wild mosquitoes.

\section{Pfin6 linkage with $k d r$ and malaria transmission}

The observed linkage reported here between $P$. falciparum susceptibility and insecticide resistance, and observations in other recent studies using different methodological approaches show the same direction of association [26, 27]. The functional analysis of para suggests that para itself is not responsible for the malaria susceptibility phenotype, although an effect in nature cannot be ruled out. The influence of transcript loss on protein abundance could not be measured because of the lack of existing antibodies against this highly hydrophobic protein. Protein abundance is often safely ignored in functional genomic experiments that produce a detectable phenotype, although in the case of para the absence of a transcript depletion phenotype for either infection or mortality means that the unknown protein level remains a caveat.

In principle, wild $k d r$-bearing mosquitoes could contribute disproportionately to malaria transmission if they live longer than wild-type mosquitoes, although the comparative lifespan of $k d r$ mosquitoes in field populations is not known. Here, mosquitoes were not exposed to insecticide treatment, and all individuals were offered a single blood meal and dissected 7-8 days afterwards, so any $k d r$-based differences in longevity, if they exist, do not influence the results.

Loss of para transcript by gene silencing did not result in higher mosquito mortality. This viability result is consistent with observations from the para orthologue in Drosophila (FlyBase ID FBgn0264255), which harbours 
many described mutations, some lethal but many viable [53], often displaying temperature-sensitive behavioural defects such as excitability that have been used as models for neurological disorders $[54,55]$. Based on the finding that mosquitoes depleted for para transcript are viable, it would be interesting to assay para-silenced mosquitoes for influence on other behavioral phenotypes that could be related to insecticide response.

\section{Conclusion}

It is a worrisome observation that mosquitoes genetically resistant to the pyrethroid insecticides commonly used on insecticide-treated bed nets may also be more susceptible to P. falciparum, and thus could potentially be more efficient malaria vectors. This effect might partially compensate for lower rates of human contact with mosquitoes after bed net distributions, and could be related to several puzzling reports of unexpected levels of malaria despite vector-control interventions [56-58].

\section{Additional files}

Additional file 1: Table S1. Catalogue of polymorphic SNPs used in the study.

Additional file 2: Figure S1. Verification of gene silencing of the ClipC9 and para genes by treatment of mosquitoes with specific dsRNAs. Transcript detected by RT-PCR is indicated to the left of the gel image for ClipC9 and to the right of the gel for para. Detection of mRNA for ribosomal protein S7 (rpS7) was used as an internal reference.

Additional file 3: Table S2. Annotated coding sequences within the haplotype carrying the Pfin 6 locus and para gene.

\section{Authors' contributions}

CM, KM, NS, KDV, and MMR conceived and designed the experiments; CM, WMG, KE, IH, AG, and MMR performed the experiments; $C M, K M, E B, K E, I H, A G$, and MMR collected and analysed the data; CM, KM, KDV, and MMR wrote the paper. All authors read and approved the final manuscript.

\section{Author details \\ ${ }^{1}$ Unit of Insect Vector Genetics and Genomics, Department of Parasites and Insect Vectors, CNRS Unit of Hosts, Vectors and Pathogens (URA3012), Lab GGIV, Institut Pasteur, 28 rue du Dr Roux, 75015 Paris, France. ${ }^{2}$ Program in Genomics, Boston Children's Hospital, Harvard Medical School, Boston, MA 02115, USA. ${ }^{3}$ Centre National de Recherche et de Formation sur le Paludisme, 01 BP 2208, Ouagadougou, Burkina Faso. ${ }^{4}$ Department of Microbiology, University of Minnesota, Saint Paul, MN 55108, USA.}

\section{Acknowledgements}

We thank Xuanzhong Li for assistance with bioinformatic analysis. We thank the CEPIA platform of the Institut Pasteur and Emma Brito-Fravallo for rearing and infecting mosquitoes. This work received financial support to KDV from the European Commission, FP7 Infrastructures \#228421 Infravec; European Research Council, Support for frontier research, Advanced Grant \#323173; National Institutes of Health, NIAID R01 \#AI073685; and French Laboratoire d'Excellence "Integrative Biology of Emerging Infectious Diseases" \#ANR-10LABX-62-IBEID. The funders had no role in study design, data collection and analysis, decision to publish, or preparation of the manuscript. Chip hybridization was performed at the BCH IDDRC Molecular Genetics Core Facility at Children's Hospital Boston supported by NIH-P30-HD18655.

\section{Compliance with ethical guidelines}

\section{Competing interests}

The authors declare they have no competing interests.

Received: 22 June 2015 Accepted: 29 September 2015

Published online: 06 October 2015

\section{References}

1. Githeko AK, Service MW, Mbogo CM, Atieli FK. Resting behaviour, ecology and genetics of malaria vectors in large scale agricultural areas of Western Kenya. Parassitologia. 1996;38:481-9.

2. Martinez-Torres D, Chandre F, Williamson MS, Darriet F, Berge JB, Devonshire AL, et al. Molecular characterization of pyrethroid knockdown resistance $(\mathrm{kdr})$ in the major malaria vector Anopheles gambiae s.s. Insect Mol Biol. 1998;7:179-84.

3. Riehle MM, Markianos K, Niare O, Xu J, Li J, Toure AM, et al. Natural malaria infection in Anopheles gambiae is regulated by a single genomic control region. Science. 2006;312:577-9.

4. Takken W, Verhulst NO. Host preferences of blood-feeding mosquitoes. Annu Rev Entomol. 2013;58:433-53.

5. Menge DM, Zhong D, Guda T, Gouagna LC, Githure JI, Beier J, et al. Quantitative trait loci controlling refractoriness to Plasmodium falciparum in natural Anopheles gambiae from a malaria endemic region in Western Kenya. Genetics. 2006;173:1337-45.

6. Riehle MM, Markianos K, Lambrechts L, Xia A, Sharakhov I, Koella JC, et al. A major genetic locus controlling natural Plasmodium falciparum infection is shared by East and West African Anopheles gambiae. Malar J. 2007;6:87.

7. Collins FH, Sakai RK, Vernick KD, Paskewitz S, Seeley DC, Miller LH, et al. Genetic selection of a Plasmodium-refractory strain of the malaria vector Anopheles gambiae. Science. 1986;234:607-10.

8. Harris C, Lambrechts L, Rousset F, Abate L, Nsango SE, Fontenille D, et al. Polymorphisms in Anopheles gambiae immune genes associated with natural resistance to Plasmodium falciparum. PLoS Pathog. 2010;6:e1001112.

9. Horton AA, Lee Y, Coulibaly CA, Rashbrook VK, Cornel AJ, Lanzaro GC, et al. Identification of three single nucleotide polymorphisms in Anopheles gambiae immune signaling genes that are associated with natural Plasmodium falciparum infection. Malar J. 2010;9:160.

10. Ffrench-Constant $\mathrm{RH}$. The molecular genetics of insecticide resistance. Genetics. 2013;194:807-15.

11. Miyazaki M, Ohyama K, Dunlap DY, Matsumura F. Cloning and sequencing of the para-type sodium channel gene from susceptible and kdrresistant German cockroaches (Blattella germanica) and house fly (Musca domestica). Mol Gen Genet. 1996;252:61-8.

12. Williamson MS, Martinez-Torres D, Hick CA, Devonshire AL. Identification of mutations in the housefly para-type sodium channel gene associated with knockdown resistance $(k d r)$ to pyrethroid insecticides. Mol Gen Genet. 1996;252:51-60.

13. Jones CM, Liyanapathirana M, Agossa FR, Weetman D, Ranson H, Donnelly MJ, et al. Footprints of positive selection associated with a mutation (N1575Y) in the voltage-gated sodium channel of Anopheles gambiae. Proc Natl Acad Sci USA. 2012;109:6614-9.

14. Pinto J, Lynd A, Vicente JL, Santolamazza F, Randle NP, Gentile G, et al. Multiple origins of knockdown resistance mutations in the Afrotropical mosquito vector Anopheles gambiae. PLoS One. 2007;2:e1243.

15. Ranson H, Jensen B, Vulule JM, Wang X, Hemingway J, Collins FH. Identification of a point mutation in the voltage-gated sodium channel gene of Kenyan Anopheles gambiae associated with resistance to DDT and pyrethroids. Insect Mol Biol. 2000;9:491-7.

16. Kabula B, Kisinza W, Tungu P, Ndege C, Batengana B, Kollo D, et al. Cooccurrence and distribution of East (L1014S) and West (L1014F) African knock-down resistance in Anopheles gambiae sensu lato population of Tanzania. Trop Med Int Health. 2014;19:331-41.

17. Namountougou M, Diabate A, Etang J, Bass C, Sawadogo SP, Gnankinie $\mathrm{O}$, et al. First report of the L1014S kdr mutation in wild populations of Anopheles gambiae M and S molecular forms in Burkina Faso (West Africa). Acta Trop. 2013;125:123-7. 
18. Djegbe I, Agossa FR, Jones CM, Poupardin R, Cornelie S, Akogbeto M, et al. Molecular characterization of DDT resistance in Anopheles gambiae from Benin. Parasit Vectors. 2014;7:409.

19. Coetzee M, Hunt RH, Wilkerson R, Della Torre A, Coulibaly MB, Besansky NJ. Anopheles coluzzii and Anopheles amharicus, new members of the Anopheles gambiae complex. Zootaxa. 2013;3619:246-74.

20. Santolamazza F, Calzetta M, Etang J, Barrese E, Dia I, Caccone A, et al. Distribution of knock-down resistance mutations in Anopheles gambiae molecular forms in west and west-central Africa. Malar J. 2008;7:74.

21. Diabate A, Brengues C, Baldet T, Dabire KR, Hougard JM, Akogbeto M, et al. The spread of the Leu-Phe kdr mutation through Anopheles gambiae complex in Burkina Faso: genetic introgression and de novo phenomena. Trop Med Int Health. 2004;9:1267-73.

22. Weill M, Chandre F, Brengues C, Manguin S, Akogbeto M, Pasteur N, et al. The kdr mutation occurs in the Mopti form of Anopheles gambiae s.s. through introgression. Insect Mol Biol. 2000;9:451-5.

23. Norris LC, Main BJ, Lee Y, Collier TC, Fofana A, Cornel AJ, et al. Adaptive introgression in an African malaria mosquito coincident with the increased usage of insecticide-treated bed nets. Proc Natl Acad Sci USA. 2015;112:815-20.

24. Clarkson CS, Weetman D, Essandoh J, Yawson AE, Maslen G, Manske $M$, et al. Adaptive introgression between Anopheles sibling species eliminates a major genomic island but not reproductive isolation. Nat Commun. 2014;5:4248.

25. Lynd A, Weetman D, Barbosa S, Egyir Yawson A, Mitchell S, Pinto J, et al. Field, genetic, and modeling approaches show strong positive selection acting upon an insecticide resistance mutation in Anopheles gambiae s.s. Mol Biol Evol. 2010;27:1117-25.

26. Ndiath MO, Cailleau A, Diedhiou SM, Gaye A, Boudin C, Richard V, et al. Effects of the kdr resistance mutation on the susceptibility of wild Anopheles gambiae populations to Plasmodium falciparum: a hindrance for vector control. Malar J. 2014;13:340.

27. Alout H, Ndam NT, Sandeu MM, Djegbe I, Chandre F, Dabire RK, et al. Insecticide resistance alleles affect vector competence of Anopheles gambiae s.s. for Plasmodium falciparum field isolates. PLoS One. 2013:8:e63849.

28. Riehle MM, Guelbeogo WM, Gneme A, Eiglmeier K, Holm I, Bischoff E, et al. A cryptic subgroup of Anopheles gambiae is highly susceptible to human malaria parasites. Science. 2011;331:596-8.

29. Gneme A, Guelbeogo WM, Riehle MM, Sanou A, Traore A, Zongo S, et al. Equivalent susceptibility of Anopheles gambiae M and S molecular forms and Anopheles arabiensis to Plasmodium falciparum infection in Burkina Faso. Malar J. 2013;12:204.

30. Fanello C, Santolamazza F, della Torre A. Simultaneous identification of species and molecular forms of the Anopheles gambiae complex by PCRRFLP. Med Vet Entomol. 2002;16:461-4.

31. Niare O, Markianos K, Volz J, Oduol F, Toure A, Bagayoko M, et al. Genetic loci affecting resistance to human malaria parasites in a West African mosquito vector population. Science. 2002;298:213-6.

32. Singer VL, Jones $L$, Yue ST, Haugland RP. Characterization of PicoGreen reagent and development of a fluorescence-based solution assay for double-stranded DNA quantitation. Anal Biochem. 1997;249:228-38.

33. GenomeStudio Genotyping Module User Guide (PDF document), http:// support.illumina.com/content/dam/illumina-support/documents/ myillumina/d2c2c169-36c7-4613-89d6-bf34588a7624/genomestudio_gt_module_v1.0_ug_11319113_reva.pdf [http://support.illumina. com/content/dam/illumina-support/documents/myillumina/d2c2c16936c7-4613-89d6-bf34588a7624/genomestudio_gt_module_v1.0_ ug_11319113_reva.pdf].

34. Barrett JC, Fry B, Maller J, Daly MJ. Haploview: analysis and visualization of LD and haplotype maps. Bioinformatics. 2005;21:263-5.

35. Mitri C, Jacques JC, Thiery I, Riehle MM, Xu J, Bischoff E, et al. Fine pathogen discrimination within the APL1 gene family protects Anopheles gambiae against human and rodent malaria species. PLoS Pathog. 2009;5:e1000576.

36. Fisher RA. Statistical methods for research workers. Edinburgh: Oliver \& Boyd; 1954.
37. Crawford JE, Bischoff E, Garnier T, Gneme A, Eiglmeier K, Holm I, et al. Evidence for population-specific positive selection on immune genes of Anopheles gambiae. G3 (Bethesda). 2012;2:1505-19.

38. Lee Y, Marsden CD, Norris LC, Collier TC, Main BJ, Fofana A, et al. Spatiotemporal dynamics of gene flow and hybrid fitness between the $M$ and $\mathrm{S}$ forms of the malaria mosquito, Anopheles gambiae. Proc Natl Acad Sci USA. 2013;110:19854-9.

39. Price AL, Zaitlen NA, Reich D, Patterson N. New approaches to population stratification in genome-wide association studies. Nat Rev Genet. 2010;11:459-63.

40. Ligoxygakis P, Pelte N, Hoffmann JA, Reichhart JM. Activation of Drosophila Toll during fungal infection by a blood serine protease. Science. 2002;297:114-6.

41. Luo H, Rose PE, Roberts TM, Dearolf CR. The Hopscotch Jak kinase requires the Raf pathway to promote blood cell activation and differentiation in Drosophila. Mol Genet Genom. 2002;267:57-63.

42. Baton LA, Robertson A, Warr E, Strand MR, Dimopoulos G. Genomewide transcriptomic profiling of Anopheles gambiae hemocytes reveals pathogen-specific signatures upon bacterial challenge and Plasmodium berghei infection. BMC Genom. 2009;10:257.

43. Dong Y, Aguilar R, Xi Z, Warr E, Mongin E, Dimopoulos G. Anopheles gambiae immune responses to human and rodent Plasmodium parasite species. PLoS Pathog. 2006;2:e52.

44. Holm I, Lavazec C, Garnier T, Mitri C, Riehle MM, Bischoff E, et al. Diverged alleles of the Anopheles gambiae leucine-rich repeat gene APL1A display distinct protective profiles against Plasmodium falciparum. PLoS One. 2012; 7:e52684.

45. Fryxell RT, Nieman CC, Fofana A, Lee Y, Traore SF, Cornel AJ, et al. Differential Plasmodium falciparum infection of Anopheles gambiae s.s. molecular and chromosomal forms in Mali. Malar J. 2012;11:133.

46. Gneme A, Guelbeogo WM, Riehle MM, Tiono AB, Diarra A, Kabre GB, et al. Plasmodium species occurrence, temporal distribution and interaction in a child-aged population in rural Burkina Faso. Malar J. 2013;12:67.

47. Ndiath MO, Brengues C, Konate L, Sokhna C, Boudin C, Trape JF, et al. Dynamics of transmission of Plasmodium falciparum by Anopheles arabiensis and the molecular forms $\mathrm{M}$ and S of Anopheles gambiae in Dielmo, Senegal. Malar J. 2008;7:136.

48. Wondji C, Frederic S, Petrarca V, Etang J, Santolamazza F, Della Torre A, et al. Species and populations of the Anopheles gambiae complex in Cameroon with special emphasis on chromosomal and molecular forms of Anopheles gambiae s.s. J Med Entomol. 2005;42:998-1005.

49. Ndiath MO, Sougoufara S, Gaye A, Mazenot C, Konate L, Faye O, et al. Resistance to DDT and pyrethroids and increased kdr mutation frequency in An. gambiae after the implementation of permethrin-treated nets in Senegal. PLoS One. 2012;7:e31943.

50. Padonou GG, Sezonlin M, Osse R, Aizoun N, Oke-Agbo F, Oussou O, et al. Impact of 3 years of large scale Indoor Residual Spraying (IRS) and Insecticide Treated Nets (ITNs) interventions on insecticide resistance in Anopheles gambiae s.l. in Benin. Parasit Vectors. 2012;5:72.

51. Rottschaefer SM, Riehle MM, Coulibaly B, Sacko M, Niare O, Morlais I, et al. Exceptional diversity, maintenance of polymorphism, and recent directional selection on the APL1 malaria resistance genes of Anopheles gambiae. PLoS Biol. 2011;9:e1000600.

52. White BJ, Lawniczak MK, Cheng C, Coulibaly MB, Wilson MD, Sagnon $\mathrm{N}$, et al. Adaptive divergence between incipient species of Anopheles gambiae increases resistance to Plasmodium. Proc Natl Acad Sci USA. 2011;108:244-9.

53. Gene Dmel/para. http://flybase.org/reports/FBgn0264255.html. Last accessed 20 Dec 2014. [http://flybase.org/reports/FBgn0264255.html]

54. Parker L, Padilla M, Du Y, Dong K, Tanouye MA. Drosophila as a model for epilepsy: bss is a gain-of-function mutation in the para sodium channel gene that leads to seizures. Genetics. 2011;187:523-34.

55. Stone B, Evans L, Coleman J, Kuebler D. Genetic and pharmacological manipulations that alter metabolism suppress seizure-like activity in Drosophila. Brain Res. 2013;1496:94-103. 
56. Coulibaly D, Travassos MA, Kone AK, Tolo Y, Laurens MB, Traore K, et al. Stable malaria incidence despite scaling up control strategies in a malaria vaccine-testing site in Mali. Malar J. 2014;13:374.

57. Trape JF, Tall A, Diagne N, Ndiath O, Ly AB, Faye J, et al. Malaria morbidity and pyrethroid resistance after the introduction of insecticide-treated bednets and artemisinin-based combination therapies: a longitudinal study. Lancet Infect Dis. 2011;11:925-32.

58. Zhou G, Afrane YA, Vardo-Zalik AM, Atieli H, Zhong D, Wamae P, Himeidan YE, et al. Changing patterns of malaria epidemiology between 2002 and 2010 in Western Kenya: the fall and rise of malaria. PLoS One. 2011;6:e20318.
Submit your next manuscript to BioMed Central and take full advantage of:

- Convenient online submission

- Thorough peer review

- No space constraints or color figure charges

- Immediate publication on acceptance

- Inclusion in PubMed, CAS, Scopus and Google Scholar

- Research which is freely available for redistribution

Submit your manuscript at www.biomedcentral.com/submit

( ) Biomed Central 